\title{
Perceived parenting threats and opportunities of Iranian mothers with breast cancer: a qualitative study
}

\author{
EFFAT MAZAHERI ${ }^{1, A-F}$, LEILA VALIZADEH ${ }^{2, A, c, D}$, VAHID ZAMANZADEH ${ }^{2, A, c, ~ D, ~}$ \\ AKRAM GHAHRAMANIAN ${ }^{3, A, c-G}$, NASRIN FOLADI 4, A, D, FARYA PHILLIPS 5, D, E \\ ORCID ID: 0000-0003-3925-1272
}

\author{
${ }^{1}$ Students' Research Committee, Tabriz University of Medical Sciences, Tabriz, Iran \\ ${ }^{2}$ Nursing and Midwifery Faculty, Tabriz University of Medical Sciences, Tabriz, Iran \\ ${ }^{3}$ Nursing and Midwifery Faculty, Hematology and Oncology Research Center, Tabriz University of Medical Sciences, \\ Tabriz, Iran \\ ${ }^{4}$ Department of Community Medicine, Ardebil University of Medical Sciences, Ardebil, Iran \\ ${ }^{5}$ Department of Health Social Work, Steve Hicks School of Social Work, the University of Texas at Austin, Texas, USA
}

A - Study Design, B - Data Collection, C - Statistical Analysis, D - Data Interpretation, E - Manuscript Preparation, F - Literature Search, G - Funds Collection

\begin{abstract}
Summary Background. A cancer diagnosis may threaten a mother's parental role, undermining her ability to care for her children and maintain family cohesion.

Objectives. The aim of this study was to explore the perceived threats to the parenting role and opportunities for growth among Iranian mothers with breast cancer.

Material and methods. This qualitative study involved single occasion, semi-structured interviews with mothers diagnosed with breast cancer $(n=20)$ who had a child younger than 16 years of age. The data was collected from May 2018 to January 2019 and analyzed using a conventional content analysis method described by Graneheim and Lundman. The MAXQDA version 10 software was used for data management.

Results. Themes from patient interviews were categorized within three domains: 1) Threats to parenting role, 2) Burden on children's life, and 3) Turning threats into opportunity: moving towards reorganization. The first domain included the categories of "physical limitations due to illness", and "psychological burden of the disease". The second domain included the categories of "children's unmet need", "children's fears and concerns", "reduced social interactions" and "failure in education". The third domain consisted of two categories, "seeking support from others" and "positive changes in family functioning".

Conclusions. Healthcare professionals should develop and implement crucial educational and counseling programs to support mothers with breast cancer. Interventions should focus on helping mothers turn perceived threats into opportunities for improving quality of life and resiliency in their family.

Key words: breast neoplasm, neoplasms, parenting, Iran.
\end{abstract}

Mazaheri E, Valizadeh L, Zamanzadeh V, Ghahramanian A, Foladi N, Farya Phillips. Perceived parenting threats and opportunities of Iranian mothers with breast cancer: a qualitative study. Fam Med Prim Care Rev 2021; 23(2): 190-195, doi: https://doi.org/10.5114/ fmpcr.2021.105922.

\section{Background}

According to estimates of the World Health Organization (WHO), breast cancer (BC) is the most common cancer diagnosed among women in the world [1]. It has also been reported as the $5^{\text {th }}$ leading cause of death in Iranian women. Studies indicate that the age of developing BC in Iranian women is ten years younger compared to developed countries [2-4]. In Iran, many women have children under the age of 18 at the time of diagnosis, and having a dependent child is one of the challenges for mothers with BC $[5,6]$. 'Mothering' is the act of a woman's emotional and physical care for her dependent child [7]. In the social and cultural context of many parts of the world, including Iran, and due to the importance of the mother's role, developing $B C$ is associated with a great deal of distress and difficulties for these mothers [8].

Maternal parenting may be threatened by the disease and through the impaired ability of a mother to care for their child [7]. A breast cancer diagnosis can threaten the mother's sense of self efficacy, maternal role and identity [9] and lead to a crisis and disruption in family dynamics, structures and routines [10]. Moreover, because of Iran's socioeconomic status and the important role of women as mothers and primary caregivers to children and extended family members, BC significantly disrupts the family's ability to function [11]. Mothers are key to the emotional well-being of families in the Middle East. Thus, they have the potential to transform traumatic events, such as a cancer diagnosis, into opportunities for growth and positive change in their family's lives [12].

The purpose of the family system is to maintain stability through adaptation to internal and external stressors that are created by change [13]. Family systems increase in complexity over time, evolving to allow greater adaptability, tolerance to change and growth by differentiation. When one member is affected by a health event, the whole family is affected differently by this change in balance [14]. Therefore, cancer may produce profound changes, not only in patients but also in the entire family system, and both the patient and family can experience growth after experiencing the illness [15]. Although several studies have explored communication and other aspects of mother-child relations in regard to mothers with $B C$, few studies describe the real world experiences of mothers in non-western countries [16]. 


\section{Objectives}

The aim of the study was to address the significant gap in literature by exploring the perceived threats to the parenting role and opportunities for growth among Iranian mothers with breast cancer.

\section{Material and methods}

\section{Study design and participants}

A qualitative study using a conventional content analysis approach was used to describe the experience of mothers diagnosed with breast cancer caring for children younger than 16 years of age. Participants were referred from the oncology clinic of Shahid Ghazi Tabatabai Hospital in East Azerbaijan Province, located in the northwest of Iran, and interviews were conducted from May 2018 to January 2019.

The participants were 20 women within the first year of diagnosis. All participants were Iranian and ranged in age from 33-49 years. Inclusion criteria consisted of women who had completed their initial course of treatment for breast cancer within the last year; who had no sign of recurrence; who had at least one child 16 years of age or younger living in the home; and who were able to speak Turkish or Persian languages. More details about the participants are presented in Table 1.

\begin{tabular}{|l|l|l|}
\hline \multicolumn{3}{|l|}{ Table 1. Socio-demographic characteristics of participants } \\
\hline Characteristics & Subgroups & Number (\%) \\
\hline Age (years) & $30-35$ & $1(5)$ \\
Mean (SD) =41.8 (4.38) & $36-45$ & $14(70)$ \\
& $\geq 46$ & $5(25)$ \\
\hline Marital status & Married & $15(75)$ \\
& Widow & $5(25)$ \\
\hline Education & Illiterate & $2(10)$ \\
& Less than high school & $8(40)$ \\
& High school & $1(5)$ \\
& Diploma & $4(20)$ \\
& University graduate & $5(25)$ \\
\hline Employment status & Homemaker & $13(65)$ \\
& Employed & $7(35)$ \\
\hline $\begin{array}{l}\text { Number of children } \\
\text { younger than 16 years }\end{array}$ & 1 & $10(50)$ \\
of age & 2 & $8(40)$ \\
\hline Duration of illness & 3 & $2(10)$ \\
(month) & $\leq 6$ & $4(20)$ \\
\hline Medical treatment & $7-12$ & $16(80)$ \\
\hline & Chemotherapy, sur- & \\
& gery and radiation & $14(70)$ \\
& Chemotherapy and & $6(30)$ \\
\hline surgery & $6(20)$
\end{tabular}

\section{Data collection}

Single occasion, semi-structured, face-to-face interviews were conducted by the primary researcher, who is a Ph.D. candidate and has the necessary academic training in conducting qualitative research. Interviews were conducted in the oncology clinic or another private location of the participants' choice, with duration times ranging from 30 to 84 minutes. The interviewer asked a number of open-ended questions at the beginning of each interview. In order to obtain rich data, probing methods (e.g. asking for elaboration; asking to give examples and reflection on the participant's quotes) were used. The initial interview questions evolved and were refined as data collection progressed and themes began to emerge. For example, with the emergence of the third category, we added the question: "How did you use the situation following the illness to improve fam- ily functioning?" In qualitative studies, the ongoing process of questioning is an integral part of understanding the unfolding lives and perspectives of participants. The process of generating and refining questions is critical to designing an effective study [17]. Data saturation was reached after 20 interviews. The interview schedule is provided in Table 2 . Persian was the main data collection language, with some use of Azari expressions. The primary researcher is fluent in both languages. Field notes were kept of each encounter with a participant.

\section{Table 2. Interview guide}

\section{Main questions}

Can you tell me about your mothering experience since the illness?

What changes have occurred in your activities since the illness? What factors, if any, have affected your ability to perform mothering duties?

How have you managed your mothering duties since the illness?

How do family members and relatives help you with your mothering duties, if at all?

How do the family members cope with your situation, and how do they respond?

As a mother, how did you use the situation following the illness to improve family functioning?

What do you do to complete your mothering duties?

What changes have occurred in the condition of children and families after the illness?

If you need help, which institutions (public and private or charitable) do you ask for help?

What kind of help do these institutions give you, and what problems do you have in getting help from them?

What experiences did you obtain after being diagnosed with the disease that have helped you to perform your motherly duties?

\section{Data analysis}

A conventional content analysis was used to code the transcribed interview data based on methods described by Graneheim and Lundman [18]. Interviews were transcribed and verified for accuracy prior to analysis. All transcripts were read several times to appreciate the meaning of the data as a whole. The initial interpretation of the data began by identifying the semantic units; open coding was then done using in-vivo and in-vitro codes after determining the semantic units. The open codes were analyzed and compared, and the coded semantic units were categorized in a category if identified as having one common element. During the coding process, constant comparative analysis was carried out in order to obtain maximum consistency within the categories and to maximize distinctions between categories. Eventually, this process led to the emergence of the three major domains [18]. MAXQDA Analytics Pro [19] version 10 software was used for data management.

\section{Data trustworthiness}

Consistency of the study was ensured through addressing the components of credibility, confirmability and dependability as outlined by Lincoln and Guba [20]. The credibility was enhanced through member checking ( 2 people), peer checking ( 2 people) and by the ongoing presence of the principal investigator in the research setting ( 8 months). The research process was carefully documented in an audit trail to increase the confirmability of the results. Two qualitative research experts examined the analysis process to meet the criterion of dependability.

\section{Ethical consideration}

This study was conducted as part of a Ph.D. nursing dissertation, and the study protocol was approved by the eth- 
ics committee of Tabriz University of Medical Sciences (code IR.TBZMED.1396.1253).

\section{Results}

\section{Sample characteristics}

As shown in Table 1, the participants were made up of 20 women (15 married, 5 widowed) within one year of diagnosis. All participants were Iranian and ranged in age from 33-49 years $(M=41.8) .70 \%$ of the participants were treated with surgery, chemotherapy and radiation.

Data analysis led to the emergence of three major domains, including:

1. Threats to parenting role,

2. Burden on children's lives,

3. Turning threats into opportunity: moving towards reorganization.

These domains and their categories are presented in Table 3.

\begin{tabular}{|c|c|}
\hline Domains & Categories \\
\hline \multirow[t]{2}{*}{ Threats to parenting role } & Physical limitations due to illness \\
\hline & Psychological burden of the disease \\
\hline \multirow[t]{4}{*}{ Burden on children's lives } & Children's unmet needs \\
\hline & Children's fears and concerns \\
\hline & Reduced social interactions \\
\hline & Failure in education \\
\hline \multirow{2}{*}{$\begin{array}{l}\text { Turning threats into op- } \\
\text { portunity: moving towards } \\
\text { reorganization }\end{array}$} & Seeking support from others \\
\hline & $\begin{array}{l}\text { Positive changes in family function- } \\
\text { ing }\end{array}$ \\
\hline
\end{tabular}

\section{Threats to parenting role}

This domain contains two categories:

1) physical limitations due to illness,

2) psychological burden of the disease.

\subsection{Physical limitations due to illness}

Physical limitations included: inadequate physical strength, lack of motor ability and inability to use their hands. The participants stated they were no longer able to care for their child/ /children.

"I used to drive my kids to school before..., but now, I can't drive much because of the swelling in my hands. I get tired quickly, and I have little ability to do things" (p. 9).

1.2. Psychological burden of the disease

The participants experienced the psychological burden of the disease, including feelings of being unable to play their mothering role, fears of lack of time left as a parent and self-doubt about their physical and mental capacity. These negative thoughts led to mothers feeling a threat to their role as a parent and caregiver.

"Annoying thoughts kept bothering me, the thought that I might get worse and couldn't do my things for the kids and not have the energy required to be a good mother" (p. 3).

\section{Burden on children's lives}

Mothers worried about the consequences of cancer on their children's lives. This domain included four categories:

1) Children's unmet needs,

2) Children's fears and concerns,

3) Reduced social interactions,

4) Failure in education.

2.1. Children's unmet needs

The mothers' concerns included their children's nutrition, cleanliness, lack of affection and lack of knowledge about can- cer. In this study, the emotional strain and physical inabilities of a life-threatening diagnosis and complication of medical treatments (arm edema and fatigue) led to a disruption in household routines and had a negative impact on family life. The children were seen as especially vulnerable to these changes, as they depend on their mother's support and care.

"I could not regularly check my children's diet and cleanliness, and frequent medical visits were a barrier to talking to my children, answering their questions and sympathizing with them" (p. 15).

\subsection{Children's fears and concerns}

The mothers reported that the physical changes due to illness had caused their children to express fears about their mother's bald heads and the possibility of dying. Mothers felt responsible for their children's growing fears and felt their illness was an undue burden on their children. The mother's cancer diagnosis was a burden not only for the mother but also for the child

"My little boy looks very worried... I've lost three kilos since I got sick; after the illness, I was always involved with the doctor and the treatment and couldn't spend much time with my kids" (p. 4).

"My young son is worried that I might die and leave him alone" (p. 7).

"While I was sick, part of my eldest daughter's hair turned white, and I know that she is under a lot of nervous pressure, but she tries not to show it" (p. 17).

\subsection{Reduced social interactions}

Without mothers being able to accompany children to their normal social activities, children's participation in recreational activities and social interactions declined a great deal. The mothers expressed that their children's interactions with other people were limited.

"We've been home with the kids most of the time since I got sick. My kids were very young ( 5 and 6 years old) and needed to play with their peers, but I couldn't go out, and my kids have not socialized much..." (p. 2).

2.4. Failure in education

Mothers whose children experienced a loss of academic motivation, academic failures or school dropouts during their illness considered themselves responsible for such things. In some cases, the practical and psychological effects of their mother's cancer were the cause of academic hardships for the children. Some even dropped out of school and felt a responsibility to help with family expenses.

"My eldest son, who is 14 years old, couldn't continue his education; my illness and the absence of his father, the heavy costs of my medication... He worked in a car repair shop to help with family expenses" (p. 17).

\section{Turning threats into opportunity: moving towards reor-} ganization

This domain is composed of the following two categories:

1) Seeking support from others,

2) Positive changes in family functioning, which showed that mothers were also capable of using the strengths of their family, relatives and social resources to reorganize the family unit and to turn the threats caused by cancer into opportunities.

3.1. Seeking support from others

The results showed that the mothers realized that they alone were unable to maintain their family's routines, but by leveraging other family members, acquaintances and social support resources, they could maintain strong family functioning. Mothers strove to guide their family, help them deal with the crisis and rearrange roles and responsibilities. Examples of such activities included reframing the mother's parenting role, balancing household tasks, utilizing formal support resources and accessing existing social support in their community. The mothers led this reorganization by considering how they could 
restore their caregiving role by saving their energy for nurturing their children rather than merely completing household tasks. Therefore, the mothers were motivated to find ways to maintain their role as a parent, meet their children's needs and prevent further emotional and physical damage to their family. They were determined to turn these threats into an opportunity for restoring family functioning through the reorganization of roles and responsibilities.

"I could not let my family become more disrupted by the illness. I tried to organize my life with the help of my children and those around me. My daughter felt very responsible... my two sisters helped me...My husband seems to feel more responsibility in this regard and takes care of the children's education" (p. 19).

Most of the participants pointed out the importance of formal social support resources. These resources include insurance systems and charities offering financial support for healthcare costs. Participants stated that although they are grateful these resources exist, they are very limited and lack any formal structure in Iran.

"We are insured, and it helps me and my family in paying some of the healthcare costs; but unfortunately, charities only donate a little money sometimes, and they have no planned training and supporting structure" (p. 11).

3.2. Positive changes in family functioning

The results showed that efforts made by mothers to access social support fostered positive family functioning in spite of the cancer and motivated their family to maintain balance as they strove to become independent again. This category includes the following three sub-categories:

1) Striving toward balance,

2) Striving toward independence,

3) Positive perspectives in the family.

3.2.1. Striving toward balance

The mothers reported that family members were now more sensitive and responsive to each other's needs. They saw how distribution of responsibilities could strengthen the family's ability to endure crisis and maintain balance.

"After the experience of illness, my family members and I became more important to each other, and we felt more responsible for each other's feelings and requests, and considering the problems we experienced, we now try not to only focus on our own tasks" (p. 14).

\subsubsection{Striving toward independence}

The participants indicated the importance of striving toward independence once treatment was over. The participants referred to the positive effects of the disease on their confidence, enabling them to cope with problems. They now felt confident the family could again become independent by learning to redistribute tasks, and they no longer needing to rely on outside help.

"We all decided not to rely on the help of others to do the housework, and we tried to coordinate the family situation without the help of other people" (p. 17).

\subsubsection{Positive perspectives in family}

The mothers seemed to maintain a positive perspective when reflecting on their family's ability to grow from their experience with cancer, increasing their appreciation for each other and strengthening their relationships. Driven by a motivation to maintain their parenting role, the mothers worked to create a supportive and successful culture within the family. Therefore, the mothers used their experience with cancer as a growth opportunity for the family and as a stimulus to appreciate their lives with their children and extended family. The struggle allowed them to appreciate the love and support of family and friends.

"Cancer has changed many things in our lives. I believe in my own ability, because I had many problems before; and now, from this experience, my family and I can grow, we can save our lives. The disease has brought us closer together. Cancer made me want to appreciate my life and my children even more" (p. 17).

\section{Discussion}

This qualitative study identified the perceived threats of Iranian mothers with breast cancer in caring for their children and explored how these mothers were able to turn these threats into opportunities for growth. The participants identified threats caused by cancer in their ability to care for their children, such as physical limitations and the psychological burden of the disease. These findings were similar to previous studies $[21,22]$ that found that physical changes and the complications of $B C$ treatment affected the parenting roles of mothers.

A unique aspect of our findings was the psychological pressure on these mothers due to Iran's social norms. Mothers are often solely responsible for all household tasks and the caregiving needs of the children and other family members. In addition, in Iranian culture, it is common for children to only engage in social activities outside of the home if accompanied by their mother; therefore, social activities and interaction outside of the home were extremely limited due to the cancer. Consequently, mothers diagnosed with breast cancer who were unable to fulfill these caregiving responsibilities caused a huge impact on the family's ability to function as, well as affecting their own identity as a mother. There were limited resources that mothers could access for support when their cancer left them physically and emotionally unable to perform their household responsibilities, and this was shown in other studies on this population in similar cultures $[23,24]$.

The mothers reported that their children had fears about the physical changes caused by cancer treatment and feared their mother would die due to the illness. Studies [25-27] conducted within the Iranian socio-cultural context showed that cancer-related taboos are a common issue that affects the perceived threats of cancer in patients and their family members. Iranians believe that cancer equals death, pain and loss of function. Iranian family members also avoid using the word cancer in front of the patient. They may attempt to hide the illness from others, and in some cases, due to changes in a patient's appearance, families reduce their interaction with others [25].

The daughter's seemed to take on more household responsibilities, and in some cases, the children had difficulties managing their own emotions and were negatively affected by the changes at home, in school and in their social life. These findings are well support by previous studies $[28,29]$. Failure in education was also especially reported in families with low economic status and single parent households. Children often dropped out of school due to the pressure of needing to financially support their family through their mother's illness. This was also seen in other studies in the Middle East, where societal nets are not widely available for families [23].

Once the mothers became aware of the threats to their family and their children's well-being due to their limited caregiving ability, the participants made efforts to redistribute roles and responsibilities within their family unit. They began to use internal and external resources and made every attempt to bring their family's level of functioning back to pre-illness levels. This shift in family member's roles and responsibilities was also reported by Phillips and Lewis in their inquiry into the impact of parental cancer [29]. These shifts in roles are not commonly accepted in Iranian culture, but since the cohesiveness of the family is extremely important, the mothers were highly motivated to find a way to balance their family's needs and limit the negative effects of their illness on their children. In particular, the eldest daughters of the family helped to maintain normal family routines. Majadi Hezaveh and Khademi [30] also found that the whole family agreed that they had to accept responsibilities beyond their typical roles in a situation where they feared the loss of a loved one.

Although some participants reported having access to public social support organizations that helped with healthcare costs, most families, especially low-income families, did not 
have access or did not realize how to fully utilize these services. Charitable services offering financial support lacked systematic structures and formal programs to adequately support family needs. In addition, it was clear that Iranian cancer patients and their families suffered from a lack of safety networks and psychological support from medical staff, which indicates the need for awareness and training in this regard [31]. A number of studies also suggest that Iranian cancer parents do not have access to recommended support services offered by professionals [32, 33].

Finally, several areas of positive growth and change from the reorganization of family values due to the experience with cancer were identified by the participants. In Iranian culture, children often live with their families until marriage and are more psychologically prepared to accept responsibility, especially in critical cases. Therefore, the opportunity created in the family following the mothers' illness can be seen as an opportunity for children to become independent. Cultural influences have been found to factor into an individual's ability to experience posttraumatic growth [34, 35].

Thus, the reorganization of the family structure that was necessary due to the illness ultimately helped the family gain balance while allowing the ill mother to maintain some of her parenting roles. This new division of duties led to positive family interactions, which were consistent with other studies [15, 36].

According to the results of this study, mothers showed increased confidence in their ability to cope with problems and considered this a positive aspect of their experience with cancer. They also reported strengthened relationships among family members and success at creating a supportive environment for their children and family. Moreover, the mothers were able to reframe the threats to their family into opportunities for growth and positive change, which is often emphasized in literature $[37,38]$.

\section{Limitations of the study}

The current study has limited generalizability due to being restricted to a single institution and a sample biased primarily toward middle-class Iranian women. Additional studies exploring mothers' experience with cancer in various Middle Eastern cultures is warranted. The sample was also limited to the mothers' perspective, and the results would have been strengthened by triangulating the results with interviews with their spouses and children. Future studies need to include larger, more diverse samples of families dealing with cancer in various cultural contexts to confirm and enhance the generalizability of the findings. Finally, some interview questions were developed as the research progressed in order to explore emerging themes and categories in depth. This may have biased the responses of some participants who were asked more specific questions that did not allow for their unique experience to be fully explored.

\section{Implication for clinical practice}

1. Healthcare administrators and policy makers must be responsive to the needs of mothers with $B C$ and their families by promoting formal support networks such as patient navigators to help patients connect to support services that assist with financial and practical needs.

2. Healthcare teams should assess parental needs and make adjustments, such as scheduling follow-up care at times that do not interfere with caregiving and by offering childcare options at oncology clinics if possible.

3. Finally, clinics should provide training within interdisciplinary teams consisting of nurses, psychologists, social workers and occupational therapists to help the teams identify and assess the short- and long-term needs of survivors that influence their social and emotional well-being throughout their cancer journey.

\section{Conclusions}

Women diagnosed with $\mathrm{BC}$ with dependent children are struggling to maintain their role as mothers while simultaneously dealing with the physical and psychological burden of their disease. Our findings reinforce previous results that suggest the importance of family and peer support, a positive attitude and access to support services when a family is coping with parental cancer. Understanding how Iranian mothers gain strength from their relationships with family, peers and the community offers healthcare professionals an opportunity to have culturally sensitive services and strategies in place to foster these relationships. Moreover, healthcare professionals can offer mothers a way to connect informally with peers at treatment centers, online or through other avenues to allow for opportunities to reduce their sense of isolation and form relationships with others who are facing similar struggles as a parent with cancer. Healthcare professionals and researchers can draw on these results to inform practice and help develop culturally sensitive interventions that address the unique needs of this population while focusing on enhancing mothers' abilities to make meaning from their experience with cancer.

Acknowledgments. We would like to thank all the participants in this study, especially the participants who reviewed the findings of the study and shared their experiences in order to increase the validity of the results.

Source of funding: This work was funded by the Tabriz University of Medical Sciences (Code IR.TBZMED.REC.1396.1253). Conflicts of interest: The authors declare no conflicts of interest.

\section{References}

1. Bray F, Ferlay J, Soerjomataram I, et al. Global cancer statistics 2018: Globocan estimates of incidence and mortality worldwide for 36 cancers in 185 countries. CA Cancer J Clin 2018; 68(6): 394-424, doi: 10.3322/caac.21492.

2. Jafari-Koshki T, Schmid VJ, Mahaki B. Trends of breast cancer incidence in Iran during 2004-2008: a Bayesian space-time model. Asian Pac J Cancer Prev 2014; 15(4): 1557-1561.

3. Mousavi SM, Montazeri A, Mohagheghi MA, et al. Breast cancer in Iran: an epidemiological review. The Breast Journal 2007; 13(4): 383-391.

4. Akbari ME, Khayamzadeh $\mathrm{M}$, Khoushnevis $\mathrm{SJ}$, et al. Five and ten years survival in breast cancer patients mastectomies vs. breast conserving surgeries personal experience. Iran J Cancer Prev 2012; 1(2): 53.

5. American Cancer Society. Breast cancer facts \& figures 2019-2020. Atlanta: American Cancer Society, Inc; 2019.

6. Arès I, Lebel S, Bielajew C. The impact of motherhood on perceived stress, illness intrusiveness and fear of cancer recurrence in young breast cancer survivors over time. Psychol Health 2014; 29(6): 651-670.

7. Vallido T, Wilkes L, Carter B, et al. Mothering disrupted by illness: a narrative synthesis of qualitative research. J Adv Nurs 2010; 66(7): $1435-1445$.

8. Haddadi M, Chaldi A, Sajjadi H, et al. Relationship between occupational class and mental health in women. Journal of Social Welfare 2011; 11(40): 107-127. 
9. Fisher C, Connor MO. "Motherhood" in the context of living with breast cancer. Cancer Nurs 2012; 35(2): 157-163.

10. Helseth S, Ulfsæt N. Parenting experiences during cancer. J Adv Nurs 2005; 52(1): 38-46.

11. Akbari ME, Sayad S, Khayamadeh M, et al. Breast cancer status in Iran: statistical analysis of 3010 cases between 1998 and 2014 . Int J Breast Cancer 2017; 2017: 2481021, doi: 10.1155/2017/2481021.

12. Heidarzadeh M, Rassouli M, Mohammadi SF, et al. Posttraumatic growth and its dimensions in patients with cancer. Middle East Journal of Cancer 2014; 5(1): 23-29.

13. Kaakinen JR, Coehlo DP, Steele R, et al. Family health care nursing: theory, practice, and research. 6th ed. Philadelphia: F.A. Davis Company; 2018.

14. Stanhope M, Lancaster J. Public health nursing-e-book: population-centered health care in the community. 9th ed. 2015; Elsevier Health Sciences.

15. Kim Y, Schulz R, Carver CS. Benefit finding in the cancer caregiving experience. Psychosom Med 2007; 69(3): $283-291$.

16. Heidarzadeh M, Rassouli M, Brant JM, et al. Dimensions of posttraumatic growth in patients with cancer: a mixed method study. Cancer Nurs 2018; 41(6): 441-449.

17. Agee J. Developing qualitative research questions: a reflective process. Int J Qual Stud Educ 2009; 22(4): 431-447.

18. Graneheim UH, Lundman B. Qualitative content analysis in nursing research: concepts, procedures and measures to achieve trustworthiness. Nurse Educ Today 2004; 24(2): 105-112.

19. Knapp CA. Research in pediatric palliative care: closing the gap between what is and is not known. Am J Hosp Palliat Care 2009; 26(5): 392-398, doi: 10.1177/1049909109345147.

20. Lincoln YS, Guba EG. Naturalistic Inquiry. SAGE Publications;1985.

21. Tam K. Occupational Therapy Practice Opportunities when working with mothers with breast cancer: a pilot study [dissertation]. The University of Puget Sound; 2012.

22. Ogce $\mathrm{F}, \mathrm{Ozkan} \mathrm{S}$. Changes in functional status and physical and psychological symptoms in women receiving chemotherapy for breast cancer. Asian Pac J Cancer Prev 2008; 9(3): 449-452.

23. Al-Zaben F, Al-Amoudi SM, El-deek BS, et al. Impact of maternal breast cancer on school-aged children in Saudi Arabia. BMC Res Notes 2014; 7(1): 261

24. Kian F, Etemadi O, Bahrami F. Exploring parenting styles of insecure Iranian mothers. Early Child Dev Care 2019; 190(1): 1-8.

25. Hassankhani H, Eghtedar S, Rahmani A, et al. A qualitative study on cancer care burden: experiences of Iranian family caregivers. Holist Nurs Pract 2019; 33(1): 17-26.

26. Mohammadi SZ, Kermanshahi SM, Vanaki Z. Pity: a qualitative study on Iranian women with breast cancer. Patient Prefer Adherence 2019; 13: 21-28.

27. Hashemi-Ghasemabadi M, Taleghani F, Kohan S, et al. Living under a cloud of threat: the experience of Iranian female caregivers with a first-degree relative with breast cancer. Psychooncology 2017; 26(5): 625-631.

28. Rosedale M. Survivor loneliness of women following breast cancer. Oncol Nurs Forum 2009; 36(2): 175-183, doi: 10.1188/09.0NF.175183.29.

29. Phillips F, Lewis FM , The adolescent's experience when a parent has advanced cancer: a qualitative inquiry. Palliat Med 2015; 29(9): 851-858.

30. Majadi Hezaveh M, Khademi M. Breast cancer: a phenomenological study. J Arak Uni Med Sci 2009; 12(1): $29-39$.

31. Vanaki Z, Parsa Yekta Z, Kazemnejad AN, et al. Interpretation of support for cancer patients under chemotherapy: a qualitative research. IJPCP 2003; 9(1): 53-61.

32. Turner J, Clavarino A, Yates $P$, et al. Enhancing the supportive care of parents with advanced cancer: development of a self-directed educational manual. Eur J Cancer 2008; 44(12): 1625-1631.

33. Turner J, Clavarino A, Yates P, et al. Development of a resource for parents with advanced cancer: what do parents want? Palliat Support Care 2007; 5(2): 135-145.

34. Taku K, Cann A, Calhoun LG, et al. The factor structure of the Posttraumatic Growth Inventory: a comparison of five models using confirmatory factor analysis. J Trauma Stress 2008; 21(2): 158-164, doi: 10.1002/jts.20305.

35. Tedeschi RG, Calhoun LG. Posttraumatic growth: conceptual foundations and empirical evidence. Psychological Inquiry 2004; 15(1): $1-18$.

36. Lim JW. The role of post-traumatic growth in promoting healthy behavior for couples coping with cancer. Support Care Cancer 2019; 27(3): 829-838.

37. Annunziata MA, Muzzatti B, Surbone A. Addressing the psychosocial wellbeing of teenage children of cancer patients and survivors. Support Care Cancer 2016; 24(2): 509-511.

38. Cormio C, Romito F, Viscanti G, et al. Psychological well-being and posttraumatic growth in caregivers of cancer patients. Front Psychol 2014; 5: 1342.

Tables: 3

Figures: 0

References: 38

Received: 19.04 .2020

Reviewed: 30.04 .2020

Accepted: 10.09 .2020

Address for correspondence:

Akram Ghahramanian, PhD, Assoc. Prof.

Faculty of Nursing and Midwifery

Hematology and Oncology Research Center

Tabriz University of Medical Sciences

South Shariati Avenue

Tabriz

Iran

Tel.: +98 9123879247

E-mail: ghahramaniana@gmail.com 